\title{
Saddle Points and Pareto Points in Multiple Objective Programming
}

\author{
Matthias Ehrgott \\ Fachbereich Mathematik \\ University of Kaiserslautern \\ 67663 Kaiserslautern \\ Germany \\ e-mail: ehrgott@mathematik.uni-kl.de \\ fax: (49) 63129081 \\ Margaret M. Wiecek ${ }^{1}$ \\ Department of Mathematical Sciences \\ Clemson University \\ Clemson, S. C. 29634 \\ U. S. A. \\ e-mail: mwiecek@math.clemson.edu \\ fax: (1) 8646565230
}

${ }^{1}$ This research was partially supported by ONR Grant N00014-97-1-784 


\begin{abstract}
In this paper relationships between Pareto points and saddle points in multiple objective programming are investigated. Convex and nonconvex problems are considered and the equivalence between Pareto points and saddle points is proved in both cases. The results are based on scalarizations of multiple objective programs and related linear and augmented Lagrangian functions. Partitions of the index sets of objectives and constraints are introduced to reduce the size of the problems. The relevance of the results in the context of decision making is also discussed.
\end{abstract}

Keywords: multiple objective programs, Pareto points, saddle points, Lagrangian functions

AMS Subject Classification: 90C29, 90C26 


\section{Introduction}

Many researchers have contributed to the theory and methodology of multiple objective programming. In particular, a lot of attention has been given to the development of various conditions for Pareto solutions. Among a great deal of studies, one specific direction has been to relate Pareto solutions to saddle points. Due to their distinctive features, for many years saddle points have been of special interest in single objective nonlinear programming. Tanino [8] was one of the pioneers who extended classical Lagrangian duality and related saddle points to nonlinear convex programs with multiple objective functions. Later, many researchers worked on vector-valued Lagrangian functions and their saddle points. Valyi [13] developed saddle-point conditions for different types of approximate Pareto solutions. Van Rooyen et al. [14] constructed a Lagrangian function for scalarized multiple objective programs and developed a saddle-point condition for Pareto solutions, which is both necessary and sufficient. Saddle points for general multiple objective programs were studied by Iacob [6]. Although the literature on various types of duality in multiple objective programming is very rich, this review indicates that there are very few papers dealing specifically with saddle points. Hence, it is desirable to further examine relationships between Pareto solutions and saddle points, which is the purpose of this article.

As we examine saddle points not only for convex but also for nonconvex problems, we apply the augmented (quadratic) Lagrangian function originally proposed in single objective nonlinear programming. In fact, this augmented function as well as more general Lagrangian functions have already been used in multiple objective programming. TenHuisen and Wiecek [11] proposed a framework for developing generalized Lagrangian-type scalarizing functions for nonconvex programs. They used the augmented function to develop solution approaches to finding Pareto points for bicriteria programs [12] and multiple criteria programs [10]. A vector-valued generalized Lagrangian was recently constructed and analyzed by Singh et al. [9].

In this paper we consider the general multiple objective program $(M O P)$ :

$$
\begin{array}{ll} 
& \min f_{q}(x) \quad q=1, \ldots, Q \\
\text { subject to } & x \in F,
\end{array}
$$


where

$$
F:=\left\{x \in \mathbb{R}^{n}: g_{j}(x) \leq 0 \quad j=1, \ldots, m\right\}
$$

is the feasible set and the functions $f_{q}(x), q=1, \ldots, Q$, and $g_{j}(x), j=$ $1, \ldots, m$, are all real-valued.

Let $\mathcal{Q}:=\{1, \ldots, Q\}$ denote the index set of the objective functions and $M:=\{1, \ldots, m\}$ denote the index set of the constraints. A feasible point $\hat{x} \in F$ is called a Pareto solution (or an efficient solution) for the $(M O P)$, if there is no other point $x \in F$ such that $f_{i}(x) \leq f_{i}(\hat{x})$ for all $i \in \mathcal{Q}$ and $f(x) \neq f(\hat{x})$. If $\hat{x}$ is a Pareto point, the corresponding point $f(\hat{x})$ in the objective space is called nondominated.

We make extensive use of the scalarization of (1) introduced by Charnes and Cooper [2] and formulated for $x^{*}$, an arbitrary feasible point of the $(M O P)$ :

$$
\begin{aligned}
\min \sum_{q=1}^{Q} f_{q}(x) & \\
\text { s.t. } f_{q}(x)-f_{q}\left(x^{*}\right) & \leq 0 \quad q=1, \ldots, Q \\
x & \in F .
\end{aligned}
$$

As this scalarization depends on the point $x^{*}$, we refer to this problem as $\left(C C\left(M O P, x^{*}\right)\right.$. It is also well known that problem (2) provides a method for finding Pareto points.

Theorem 1 A point $x^{*} \in F$ is a Pareto solution for the (MOP) if and only if $x^{*}$ is an optimal solution for the problem $\left(C C\left(M O P, x^{*}\right)\right)$.

In Section 2, we partition the index set of the objective functions in order to show how to reach a Pareto solution given a feasible point that is not Pareto. We achieve this by minimizing over the objective functions whose values can be still improved while the other objectives' values do not deteriorate. We propose two partitions, one for feasible points only while in the other we allow infeasible points. The partitions of the index set of the objective functions are complemented by a partition of the index set of the constraints. These partitions determine a framework within which we study relationships between Pareto points and saddle points of convex and nonconvex multiple objective programs. In Section 3, we derive a saddle point characterization of 
Pareto points for convex programs. Although in this section we follow upon the results in [14], we introduce different index sets to define the Lagrangian functions which makes our results easier to handle. In Section 4, we analyze nonconvex programs and derive a saddle point characterization of Pareto points applying the augmented Lagrangian function. Throughout the paper we include comments on the usefulness of our results in multiple criteria decision making. Final conclusions are contained in Section 5.

\section{Partitioning the Index Set of Objective Functions}

Given a feasible point $x^{*}$ of the problem $(M O P)$ and the objective function values at this point, one may be interested whether it is still possible to improve the values of some criteria while the other criteria do not deteriorate. To answer this question, it is convenient to introduce the set of feasible points which allow improvement of some objective functions with respect to a given point $x^{*}$. Defining

$$
F^{\leq}\left(x^{*}\right):=\left\{x \in F: f_{i}(x) \leq f_{i}\left(x^{*}\right) \forall i \in \mathcal{Q}\right\}
$$

and using the concept of level sets

$$
L_{\leq}^{i}\left(x^{*}\right):=\left\{x \in F: f_{i}(x) \leq f_{i}\left(x^{*}\right)\right\},
$$

we have that $F \leq\left(x^{*}\right)$ is equal to the intersection of all the level sets at the point $x^{*}$ :

$$
F^{\leq}\left(x^{*}\right)=\bigcap_{i=1}^{Q} L_{\leq}^{i}\left(f_{i}\left(x^{*}\right)\right) .
$$

In [5] it was shown that a point $x^{*} \in F$ is a Pareto point of the $(M O P)$ if and only if

$$
\bigcap_{i=1}^{Q} L_{\leq}^{i}\left(x^{*}\right)=\bigcap_{i=1}^{Q} L_{=}^{i}\left(x^{*}\right),
$$

where $L_{=}^{i}\left(x^{*}\right)$ denotes the level curve of the objective $f_{i}$ passing through $x^{*}$. Clearly, if $x^{*}$ is not a Pareto point these intersections must be different. As it is desirable to distinguish between these objective functions that allow 
improvement and those that do not, we partition the index set $\mathcal{Q}$ with respect to $F \leq\left(x^{*}\right)$ :

$$
\mathcal{Q}^{=}\left(x^{*}\right):=\left\{i \in \mathcal{Q}: \forall x \in F^{\leq}\left(x^{*}\right) f_{i}(x)=f_{i}\left(x^{*}\right)\right\}
$$

and

$$
\mathcal{Q}^{<}\left(x^{*}\right):=\left\{i \in \mathcal{Q}: \exists x \in F^{\leq}\left(x^{*}\right) \text { such that } f_{i}(x)<f_{i}\left(x^{*}\right)\right\} .
$$

Since only the feasible points are considered in the definition of $\mathcal{Q}^{=}\left(x^{*}\right)$ and $Q^{<}\left(x^{*}\right)$, this partition will be called the feasible point partition of the index set of the objective functions.

Given a point $x^{*} \in F$ which is not a Pareto point for the $(M O P)$, we shall show that one does not have to consider all the objective functions to find a Pareto point and can restrict the optimization to the objective functions in $\mathcal{Q}^{<}\left(x^{*}\right)$. We therefore formulate a multiple objective program with a smaller number of objective functions and refer to it as $\left(\operatorname{MOP}\left(\mathcal{Q}^{<}\left(x^{*}\right)\right)\right)$, as this problem depends on the point $x^{*}$ :

$$
\begin{aligned}
& \min f_{q}(x) \quad q \in \mathcal{Q}^{<}\left(x^{*}\right) \\
& \text { subject to } \quad x \in F^{\leq}\left(x^{*}\right) .
\end{aligned}
$$

It turns out that every Pareto point of the smaller problem (4) is also Pareto for the original problem (1).

Theorem 2 If $\bar{x} \in F^{\leq}\left(x^{*}\right)$ is a Pareto point for problem $\left(\operatorname{MOP}\left(\mathcal{Q}^{<}\left(x^{*}\right)\right)\right)$ then $\bar{x}$ is also a Pareto point for the (MOP).

\section{Proof:}

Let $\bar{x}$ be a Pareto point of the problem $\left(\operatorname{MOP}\left(\mathcal{Q}^{<}\left(x^{*}\right)\right)\right)$. Assume that $\bar{x}$ is not a Pareto point for the $(M O P)$. Then there is an $x^{\prime} \in F$ such that

$$
\begin{aligned}
f_{i}\left(x^{\prime}\right) & \leq f_{i}(\bar{x}) \quad \forall i \in \mathcal{Q} \\
f_{k}\left(x^{\prime}\right) & <f_{k}(\bar{x}) \quad \text { for some } k \in \mathcal{Q} .
\end{aligned}
$$

Because $\bar{x} \in F \leq\left(x^{*}\right)$ we have also that $f_{i}(\bar{x}) \leq f_{i}\left(x^{*}\right)$ and therefore $x^{\prime} \in$ $F \leq\left(x^{*}\right)$. From $f_{k}\left(x^{\prime}\right)<f_{k}\left(x^{*}\right)$ we get $k \in \mathcal{Q}^{<}\left(x^{*}\right)$. In particular we conclude that there exists an $x^{\prime} \in F \leq\left(x^{*}\right)$ such that

$$
\begin{aligned}
f_{i}\left(x^{\prime}\right) & \leq f_{i}(\bar{x}) \quad \forall i \in \mathcal{Q}^{<}\left(x^{*}\right) \\
f_{k}\left(x^{\prime}\right) & <f_{k}(\bar{x}) \quad \text { for some } k \in \mathcal{Q}^{<}\left(x^{*}\right)
\end{aligned}
$$


which contradicts the fact that $\bar{x}$ is a Pareto solution for the smaller problem $\left(\operatorname{MOP}\left(\mathcal{Q}^{<}\left(x^{*}\right)\right)\right)$.

Corollary 1 If $x^{*}$ is a Pareto solution for the problem (MOP) then the problem $\left(\operatorname{MOP}\left(\mathcal{Q}^{<}\left(x^{*}\right)\right)\right)$ is not defined.

\section{Proof:}

If $x^{*}$ is a Pareto point for the $(M O P)$, then $F \leq\left(x^{*}\right)=\cap_{i=1}^{Q} L_{=}^{i}\left(x^{*}\right)$ which implies $\mathcal{Q}^{<}\left(x^{*}\right)=\emptyset$ (see [5]).

An analogous partition, complementing the partition of objectives, can be introduced for the index set of the constraints:

$$
M^{=}\left(x^{*}\right):=\left\{j \in M: \forall x \in F^{\leq}\left(x^{*}\right) g_{j}(x)=0\right\}
$$

and

$$
M^{<}\left(x^{*}\right):=\left\{j \in M: \exists x \in F^{\leq}\left(x^{*}\right) \text { such that } g_{j}(x)<0\right\} .
$$

Here we simply distinguish between the active and inactive constraints with respect to the set $F \leq\left(x^{*}\right)$. This partition is called the feasible point partition of the index set of the constraints.

We may ignore the feasibility requirement in the definition of $F \leq\left(x^{*}\right)$, which in some situations will lead to more useful results, as we will see later. Define

$$
F_{0}^{\leq}\left(x^{*}\right):=\left\{x \in \mathbb{R}^{n}: f_{i}(x) \leq f_{i}\left(x^{*}\right) \forall i \in \mathcal{Q}\right\} .
$$

The partition of the index set $\mathcal{Q}$ is now defined by

$$
\mathcal{Q}_{0}^{=}\left(x^{*}\right):=\left\{i \in \mathcal{Q}: \forall x \in F_{0}^{\leq}\left(x^{*}\right) f_{i}(x)=f_{i}\left(x^{*}\right)\right\}
$$

and

$$
\mathcal{Q}_{0}^{<}\left(x^{*}\right):=\left\{i \in \mathcal{Q}: \exists x \in F_{0}^{\leq}\left(x^{*}\right) \text { such that } f_{i}(x)<f_{i}\left(x^{*}\right)\right\} .
$$

Due to the fact that $F_{0}^{\leq}\left(x^{*}\right)$ may contain infeasible points, this partition of $\mathcal{Q}$ is called the infeasible point partition of the index set of the objective functions. In the view of $F_{0}^{\leq}\left(x^{*}\right)$, we can now modify the smaller problem (4) and formulate the problem $\left(\operatorname{MOP}\left(\mathcal{Q}_{0}^{<}\left(x^{*}\right)\right)\right.$ :

$$
\begin{aligned}
& \min f_{q}(x) \quad q \in \mathcal{Q}_{0}^{<}\left(x^{*}\right) \\
& \text { subject to } \quad x \in F_{0}^{\leq}\left(x^{*}\right) .
\end{aligned}
$$

We then obtain the following result. 
Theorem 3 Let $x^{*}$ be a feasible point of the $(M O P)$, which is not a Pareto point. Then if $\hat{x} \in F_{0}^{\leq}\left(x^{*}\right)$ is a Pareto point for the problem $\left(\operatorname{MOP}\left(\mathcal{Q}_{0}^{<}\left(x^{*}\right)\right)\right.$ and feasible for the $(M O P)$ then it is also a Pareto solution for the $(M O P)$.

The proof is analogous to the proof of Theorem 2 .

We now define the corresponding infeasible point partition of the index set of the constraints with respect to $F_{0}^{\leq}\left(x^{*}\right)$ :

$$
M_{0}^{=}\left(x^{*}\right):=\left\{j \in M: \forall x \in F_{0}^{\leq}\left(x^{*}\right) g_{j}(x)=0\right\}
$$

and

$$
M_{0}^{<}\left(x^{*}\right):=\left\{j \in M: \exists x \in F_{0}^{\leq}\left(x^{*}\right) \text { such that } g_{j}\left(x^{*}\right)<0\right\} .
$$

Having established the foundation for further analysis, we turn our attention to Pareto points and saddle points of convex and nonconvex problems.

\section{Convex Problems}

In this section we investigate the special case of convex multiple objective programs, i.e. we assume that all the functions $f_{i}$ and $g_{j}$ are convex in the $(M O P)$. In particular, the feasible set $F$ is convex. Note that if the $(M O P)$ is a convex problem then the problem $\left(\operatorname{MOP}\left(\mathcal{Q}^{<}\left(x^{*}\right)\right)\right)$ is also convex, because $F \leq\left(x^{*}\right)$ is the intersection of the (convex) level sets $L_{\leq}^{i}\left(x^{*}\right)$.

In Section 3.1 we use the feasible point partitions and prove a saddle point characterization of Pareto points for the convex $(M O P)$ while in Section 3.2 we present a saddle-point result based on the infeasible point partitions. Some of these results have first been obtained in [4].

\subsection{A Saddle Point Characterization for the Feasible Point Partition}

Using the feasible point partition of the index set of the objective functions defined in Section 2 the following result holds:

Lemma $1 A$ point $x^{*} \in F$ is not a Pareto point for the (MOP) if and only if $\mathcal{Q}^{<}\left(x^{*}\right) \neq \emptyset$ and there exists a point $\bar{x} \in F$ such that

$$
\begin{aligned}
& f_{i}(\bar{x})<f_{i}\left(x^{*}\right) \quad \forall i \in \mathcal{Q}^{<}\left(x^{*}\right) \\
& f_{i}(\bar{x})=f_{i}\left(x^{*}\right) \quad \forall i \in \mathcal{Q}^{=}\left(x^{*}\right)
\end{aligned}
$$




\section{Proof:}

The condition of the lemma is sufficient for $x^{*}$ not to be a Pareto point. We only show that it is necessary. Let $x^{*}$ be a point that is not Pareto. Then $\mathcal{Q}^{<}\left(x^{*}\right)$ is not empty. For every $i \in \mathcal{Q}^{<}\left(x^{*}\right)$ choose an $x^{i} \in F \leq\left(x^{*}\right)$ with $f_{i}\left(x^{i}\right)<f_{i}\left(x^{*}\right)$. Then the point $\bar{x}:=\sum_{i \in \mathcal{Q}^{<}\left(x^{*}\right)} \alpha_{i} x^{i}$, where $\alpha_{i}>0$ and $\sum \alpha_{i}=1$, has the desired properties. Due to convexity, $\bar{x}$ belongs to $F \leq\left(x^{*}\right)$. Consequently, by the definition of $\bar{x}$

$$
f_{i}(\bar{x})<f_{i}\left(x^{*}\right) \text { for all } i \in \mathcal{Q}^{<}\left(x^{*}\right)
$$

and by the definition of $\mathcal{Q}^{=}\left(x^{*}\right)$,

$$
f_{i}(\bar{x})=f_{i}\left(x^{*}\right) \text { for all } i \in \mathcal{Q}^{=}\left(x^{*}\right) .
$$

If we think of the problem $\left(M O P\left(\mathcal{Q}^{<}\left(x^{*}\right)\right)\right)$ in the context of convex problems, we can strengthen Theorem 2. If $x^{*}$ is not a Pareto point for the $(M O P)$, then by Lemma 1 there exists a Pareto point $\bar{x}$ for the problem $\left(\operatorname{MOP}\left(\mathcal{Q}^{<}\left(x^{*}\right)\right)\right)$ such that

$$
\begin{aligned}
& f_{i}(\bar{x})=f_{i}\left(x^{*}\right) \quad \forall i \in \mathcal{Q}^{=}\left(x^{*}\right) \\
& f_{i}(\bar{x})<f_{i}\left(x^{*}\right) \quad \forall i \in \mathcal{Q}^{<}\left(x^{*}\right)
\end{aligned}
$$

and furthermore, due to Theorem $2, \bar{x}$ is a Pareto point for the $(M O P)$.

Example 1 In Figure 1 on the left hand side $x^{*}$ is not a Pareto solution, $\mathcal{Q}^{=}\left(x^{*}\right)=\emptyset$ and $\mathcal{Q}^{<}\left(x^{*}\right)=\mathcal{Q}$. A convex combination of $x^{1}, x^{2}$ and $x^{3}$ yields $\bar{x}$ dominating $x^{*}$. On the right hand side, $x^{*}$ is a Pareto solution since $\mathcal{Q}^{<}\left(x^{*}\right)=\emptyset$ and $\mathcal{Q}^{=}\left(x^{*}\right)=\mathcal{Q}$.

From the geometrical characterization of Pareto solutions given by (3) we could also conclude that a point $x^{*} \in F$ is Pareto if and only if $\mathcal{Q}^{<}\left(x^{*}\right)$ is empty. (This is the same as saying that the intersection of all level sets of the point $x^{*}$ is equal to the intersection of all level curves of this point.) This conclusion is valid for general (nonconvex) problems and implies that for a point that is not a Pareto solution, the point $x^{i}$ used in the proof of Lemma 1 exists. However, the existence of the point $\bar{x}$ which satisfies all the conditions of this lemma simultaneously cannot be shown in general. 

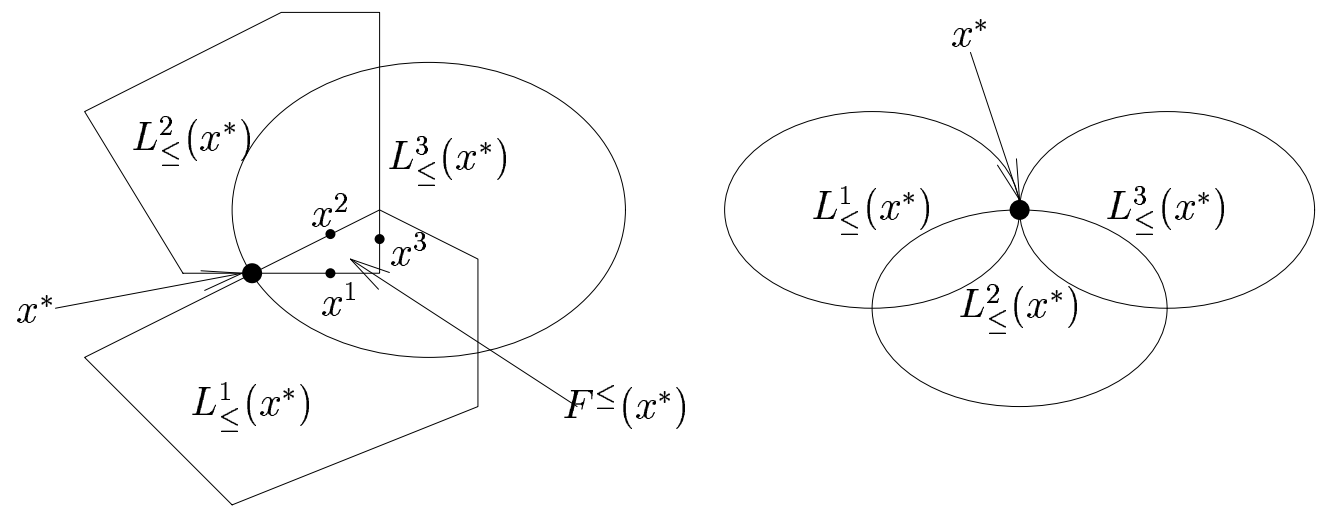

Figure 1: Illustration of Lemma 1

The result of Lemma 1 for the convex $(M O P)$ is stronger: the case that $x^{*}$ is not a Pareto point implies the existence of a feasible point $\bar{x}$ for which $f_{i}(\bar{x})<f_{i}\left(x^{*}\right)$ for all $i \in \mathcal{Q}^{<}\left(x^{*}\right)$ simultaneously. This cannot be guaranteed for nonconvex problems, i.e. in the general context of [5].

Including the feasible partition of the index set of the constraints, $M^{<}\left(x^{*}\right)$ and $M^{=}\left(x^{*}\right)$, we extend Lemma 1 and obtain a more specific result concerning the constraints.

Lemma $2 A$ point $x^{*} \in F$ is not a Pareto point for the (MOP) if and only if $\mathcal{Q}^{<}\left(x^{*}\right) \neq \emptyset$ and there exists a point $\bar{x} \in F$ such that

$$
\begin{aligned}
& f_{i}(\bar{x})<f_{i}\left(x^{*}\right) \quad \forall i \in \mathcal{Q}^{<}\left(x^{*}\right) \\
& f_{i}(\bar{x})=f_{i}\left(x^{*}\right) \quad \forall i \in \mathcal{Q}^{=}\left(x^{*}\right) \\
& g_{j}(\bar{x})<0 \quad \forall j \in M^{<}\left(x^{*}\right) \\
& g_{j}(\bar{x})=0 \quad \forall j \in M^{=}\left(x^{*}\right) .
\end{aligned}
$$

\section{Proof:}

The 'if' part of the lemma is clear. To show the 'only if' part, let $x^{*}$ be a feasible solution but not Pareto. First note that if $M^{<}\left(x^{*}\right)$ is empty, the result follows directly from the definition of $M^{=}\left(x^{*}\right)$ and Lemma 1. Otherwise for every $i \in \mathcal{Q}^{<}\left(x^{*}\right)$ choose an $\hat{x}^{i} \in F \leq\left(x^{*}\right)$ with $f_{i}\left(\hat{x}^{i}\right)<f_{i}\left(x^{*}\right)$ and for every $j \in M^{<}\left(x^{*}\right)$ choose an $\bar{x}^{j} \in F \leq\left(x^{*}\right)$ with $g_{j}\left(\bar{x}^{j}\right)<0$. 
Define $\bar{x}$ as a strict convex combination of these points,

$$
\bar{x}:=\sum_{i \in \mathcal{Q}^{<}\left(x^{*}\right)} \alpha_{i} \hat{x}^{i}+\sum_{j \in M^{<}\left(x^{*}\right)} \beta_{j} \bar{x}^{j}
$$

where $\alpha_{i}, \beta_{j}>0$ for every $i \in \mathcal{Q}^{<}\left(x^{*}\right), j \in M^{<}\left(x^{*}\right)$ and $\sum_{i \in \mathcal{Q}^{<}\left(x^{*}\right)} \alpha_{i}+$ $\sum_{j \in M<\left(x^{*}\right)} \beta_{j}=1$. Then, due to convexity of $f_{k}$ and the choice of $\hat{x}^{i}$ and $\bar{x}^{j}$, we have

$$
f_{k}(\bar{x}) \leq \sum_{i \in M^{<}\left(x^{*}\right)} \alpha_{i} f_{k}\left(\hat{x}^{i}\right)+\sum_{j \in \mathcal{Q}^{<}\left(x^{*}\right)} \beta_{j} f_{k}\left(\bar{x}^{j}\right)<f_{k}\left(x^{*}\right)
$$

for all $k \in \mathcal{Q}^{<}\left(x^{*}\right)$, i.e. (6). Furthermore, by analogous arguments for $g_{l}$ we have

$$
g_{l}(\bar{x}) \leq \sum_{i \in M<\left(x^{*}\right)} \alpha_{i} g_{l}\left(\hat{x}^{i}\right)+\sum_{j \in \mathcal{Q}<\left(x^{*}\right)} \beta_{j} g_{l}\left(\bar{x}^{j}\right)<0
$$

for all $l \in M^{<}\left(x^{*}\right)$, i.e. (8). Due to convexity of $F \leq\left(x^{*}\right), \bar{x} \in F \leq\left(x^{*}\right)$. Then by the definitions of $Q^{=}\left(x^{*}\right)$ and $M^{=}\left(x^{*}\right)$ we obtain (7) and (9).

Corollary 2 If a point $x^{*} \in F$ is a Pareto point for the $(M O P)$ and $M \neq$ $M^{=}\left(x^{*}\right)$, then there exists a point $\hat{x} \in F^{\leq}\left(x^{*}\right)$ such that (7), (8) and (9) hold.

\section{Proof:}

Observe that for every $j \in M^{<}\left(x^{*}\right)$ there exists an $x^{j} \in F^{\leq}\left(x^{*}\right)$ such that $g_{j}\left(x^{j}\right)<0$. Define $\hat{x}:=\sum_{j \in M<\left(x^{*}\right)} \alpha_{j} x^{j}$, where $\alpha_{j}>0$ and $\sum \alpha_{j}=1$. Then

$$
g_{l}(\hat{x}) \leq \sum_{j \in M<\left(x^{*}\right)} \alpha_{j} g_{j}\left(x^{*}\right)<0
$$

for all $l \in M^{<}\left(x^{*}\right)$. Since the set $F \leq\left(x^{*}\right)$ is convex, $\hat{x} \in F \leq\left(x^{*}\right)$. Therefore $g_{j}(\hat{x})=0$ for $j \in M^{=}\left(x^{*}\right)$ and $f_{i}(\hat{x})=f_{i}\left(x^{*}\right)$ for $i \in \mathcal{Q}^{=}\left(x^{*}\right)$.

Define now the set

$$
\begin{aligned}
\bar{F}^{\leq}\left(x^{*}\right):= & \left\{x \in \mathbb{R}^{n}: f_{i}(x) \leq f_{i}\left(x^{*}\right) \forall i \in \mathcal{Q}^{=}\left(x^{*}\right)\right\} \\
\cap & \left\{x \in \mathbb{R}^{n}: g_{j}(x) \leq 0 \forall j \in M^{=}\left(x^{*}\right)\right\} .
\end{aligned}
$$

and the Lagrangian function

$$
L(x, \lambda, \mu):=\sum_{i \in \mathcal{Q}^{<}\left(x^{*}\right)} \lambda_{i} f_{i}(x)+\sum_{j \in M<\left(x^{*}\right)} \mu_{j} g_{j}(x) .
$$


We now present the main result of this section showing that the existence of a saddle point of the Lagrangian function $L(x, \lambda, \mu)$ is a necessary and sufficient condition for a point $x^{*}$ to be Pareto. Lemma 2 and Corollary 2 provide the foundation for the proof of Theorem 4 which follows upon the proof of the main result in [14].

Theorem $4 A$ point $x^{*} \in F$ is a Pareto solution for the (MOP) if and only if there exist multipliers $\lambda^{*} \geq 0, \lambda^{*} \neq 0$ and $\mu^{*} \geq 0$ such that

$$
L\left(x^{*}, \lambda^{*}, \mu\right) \leq L\left(x^{*}, \lambda^{*}, \mu^{*}\right) \leq L\left(x, \lambda^{*}, \mu^{*}\right)
$$

holds for all $\mu \geq 0$ and for all $x \in \bar{F}^{\leq}\left(x^{*}\right)$.

\section{Proof:}

We first show that (11) is necessary for a point $x^{*}$ to be a Pareto point. Suppose $x^{*} \in F$ is a Pareto point. Then $x^{*} \in F \leq\left(x^{*}\right)$ that is a convex set. Assume that $\mathcal{Q}^{<}\left(x^{*}\right)=\{1, \ldots, r\}$ and $M^{<}\left(x^{*}\right)=\{1, \ldots, s\}$ and define the following two sets:

$$
K_{1}:=\left\{y \in \mathbb{R}^{r+s}: y>\left(\begin{array}{c}
f_{1}(x) \\
\cdots \\
f_{r}(x) \\
g_{1}(x) \\
\cdots \\
g_{s}(x)
\end{array}\right) \text { for at least one } x \in \bar{F}^{\leq}\left(x^{*}\right)\right\}
$$

and

$$
K_{2}:=\left\{y \in \mathbb{R}^{r+s}: y<<\left(\begin{array}{c}
f_{1}\left(x^{*}\right) \\
\cdots \\
f_{r}\left(x^{*}\right) \\
0 \\
\cdots \\
0
\end{array}\right)\right\}
$$

where $K_{2}$ is convex. To show that $K_{1}$ is convex is straightforward since all the functions $f_{i}, i=1, \ldots, r$ and $g_{j}, j=1, \ldots, s$ are convex. We now prove 
that $K_{1} \cap K_{2}=\emptyset$. Otherwise there exists some $y^{0} \in K_{1} \cap K_{2}$ such that

$$
\left(\begin{array}{c}
f_{1}\left(x^{*}\right) \\
\cdots \\
f_{r}\left(x^{*}\right) \\
0 \\
\cdots \\
0
\end{array}\right) \gg y^{0}>\left(\begin{array}{c}
f_{1}\left(x^{0}\right) \\
\cdots \\
f_{r}\left(x^{0}\right) \\
g_{1}\left(x^{0}\right) \\
\cdots \\
g_{s}\left(x^{0}\right)
\end{array}\right)
$$

for some $x^{0} \in \bar{F}^{\leq}\left(x^{*}\right)$ which implies that $x^{0} \in F$. Therefore due to Lemma $2, x^{*}$ is not a Pareto point, which is a contradiction. As the sets $K_{1}$ and $K_{2}$ are convex, there exist a separating hyperplane for $K_{1}$ and $K_{2}$ with a normal vector $\left(\lambda^{*}, \mu^{*}\right) \neq 0, \lambda^{*} \in \mathbb{R}^{r}, \mu^{*} \in \mathbb{R}^{s}$, and a scalar $\alpha \in \mathbb{R}$ such that

$$
\sum_{i=1}^{r} \lambda_{i}^{*} y_{i}^{1}+\sum_{j=1}^{s} \mu_{j}^{*} y_{j}^{1} \geq \alpha \geq \sum_{i=1}^{r} \lambda_{i}^{*} y_{i}^{2}+\sum_{j=1}^{s} \mu_{j}^{*} y_{j}^{2}
$$

for all $y^{1} \in \operatorname{cl}\left(K_{1}\right)$ and all $y^{2} \in \operatorname{cl}\left(K_{2}\right)$, where $\operatorname{cl}(S)$ denotes the closure of a set $S$. To prove (11), we choose several points $y^{1} \in \operatorname{cl}\left(K_{1}\right)$ and $y^{2} \in \operatorname{cl}\left(K_{2}\right)$ and examine (12) in each of the following steps.

Step 1: Let

$$
\begin{aligned}
y^{1} & =\left(f_{1}\left(x^{*}\right), \ldots, f_{r}\left(x^{*}\right), g_{1}\left(x^{*}\right), \ldots, g_{s}\left(x^{*}\right)\right) \\
\text { and } y^{2} & =\left(f_{1}\left(x^{*}\right), \ldots, f_{r}\left(x^{*}\right), 0, \ldots, 0\right) .
\end{aligned}
$$

Then (12) yields $L\left(x^{*}, \lambda^{*}, \mu^{*}\right) \geq \alpha \geq \sum_{i=1}^{r} \lambda_{i}^{*} f_{i}\left(x^{*}\right)=L\left(x^{*}, \lambda^{*}, 0\right) \geq$ $L\left(x^{*}, \lambda^{*}, \mu\right)$ for all $\mu \geq 0$ since $\sum_{j=1}^{s} \mu_{j} g_{j}\left(x^{*}\right) \leq 0$.

Step 2: Let

$$
\begin{aligned}
y^{1} & =\left(f_{1}(x), \ldots, f_{r}(x), g_{1}(x), \ldots, g_{s}(x)\right) \\
\text { and } y^{2} & =\left(f_{1}\left(x^{*}\right), \ldots, f_{r}\left(x^{*}\right), g_{1}\left(x^{*}\right), \ldots, g_{s}\left(x^{*}\right)\right)
\end{aligned}
$$

where $x \in \bar{F}^{\leq}\left(x^{*}\right)$. Then (12) yields $L\left(x, \lambda^{*}, \mu^{*}\right) \geq L\left(x^{*}, \lambda^{*}, \mu^{*}\right)$.

Step 3: Let

$$
\begin{aligned}
y^{1} & =\left(f_{1}\left(x^{*}\right), \ldots, f_{r}\left(x^{*}\right), 0, \ldots, 0\right) \\
\text { and } y^{2} & =\left(f_{1}\left(x^{*}\right), \ldots, f_{r}\left(x^{*}\right), y_{1}, \ldots, y_{s}\right)
\end{aligned}
$$


where $y_{j}<0$ for all $j=1, \ldots, s$.

From (12) we get $\sum_{i=1}^{r} \lambda_{i}^{*} f_{i}\left(x^{*}\right)+\sum_{j=1}^{s} \mu_{j}^{*} 0 \geq \sum_{i=1}^{r} \lambda_{i}^{*} f_{i}\left(x^{*}\right)+\sum_{j=1}^{s} \mu_{j}^{*} y_{j}$. Hence $0 \geq \sum_{j=1}^{s} \mu_{j}^{*} y_{j}$ for all such $y^{2}$ and we conclude that $\mu_{j}^{*} \geq 0$ for all $j \in M^{<}\left(x^{*}\right)$.

Step 4: Let

$$
\begin{aligned}
y^{1} & =\left(y_{1}, \ldots, y_{r}, 0, \ldots, 0\right) \\
\text { and } y^{2} & =\left(f_{1}\left(x^{*}\right), \ldots, f_{r}\left(x^{*}\right), 0, \ldots, 0\right)
\end{aligned}
$$

where $y_{i} \geq f_{i}\left(x^{*}\right)$ for $i=1, \ldots, r$. Therefore $\sum_{i=1}^{r} \lambda_{i}^{*} y_{i} \geq \sum_{i=1}^{r} \lambda_{i}^{*} f_{i}\left(x^{*}\right)$. Hence $\sum_{i=1}^{r} \lambda_{i}^{*}\left(y_{i}-f_{i}\left(x^{*}\right)\right) \geq 0$ for all such $y^{1}$ and we conclude that $\lambda_{i}^{*} \geq 0$ for all $i \in \mathcal{Q}^{<}\left(x^{*}\right)$.

Step 5: Assume that $\lambda^{*}=0$. From (12) we have $\sum_{j=1}^{s} \mu_{j}^{*}\left(y_{j}^{1}-y_{j}^{2}\right) \geq 0$ for all $y^{1} \in \operatorname{cl}\left(K_{1}\right)$ and all $y^{2} \in \operatorname{cl}\left(K_{2}\right)$. Let

$$
\begin{aligned}
y^{1} & =\left(f_{1}(\bar{x}), \ldots, f_{r}(\bar{x}), g_{1}(\bar{x}), \ldots, g_{s}(\bar{x})\right) \\
\text { and } y^{2} & =\left(f_{1}\left(x^{*}\right), \ldots, f_{r}\left(x^{*}\right), 0, \ldots, 0\right)
\end{aligned}
$$

where $\bar{x}$ is chosen as in Corollary 2, i.e. $g_{j}(\bar{x})<0$ for all $j \in M^{<}\left(x^{*}\right)$. As $\left(\lambda^{*}, \mu^{*}\right) \neq 0, \mu^{*}$ cannot be zero, and $\sum_{j=1}^{s} \mu_{j}^{*}\left(g_{j}(\bar{x})-0\right)<0$, which contradicts the expression produced by (12). Therefore $\lambda^{*} \neq 0$.

We now prove that (11) is also sufficient for $x^{*}$ to be a Pareto point. The left hand side of equation (11)

$$
\sum_{i=1}^{r} \lambda_{i}^{*} f_{i}\left(x^{*}\right)+\sum_{j=1}^{s} \mu_{j} g_{j}\left(x^{*}\right) \leq \sum_{i=1}^{r} \lambda_{i}^{*} f_{i}\left(x^{*}\right)+\sum_{j=1}^{s} \mu_{j}^{*} g_{j}\left(x^{*}\right)
$$

is equivalent to $\sum_{j=1}^{s}\left(\mu_{j}-\mu_{j}^{*}\right) g_{j}\left(x^{*}\right) \leq 0$ for all $\mu \geq 0$. This can only be true if $g_{j}\left(x^{*}\right) \leq 0$ for all $j \in M^{<}\left(x^{*}\right)$. Since by assumption $x^{*} \in \bar{F} \leq\left(x^{*}\right)$ and therefore $g_{j}\left(x^{*}\right) \leq 0$ for all $j \in M^{=}\left(x^{*}\right)$, we conclude that $x^{*}$ is feasible.

Suppose $x^{*}$ is not a Pareto point. Then by Lemma 2, there exists a point $\bar{x} \in F$ such that (6) - (9) hold. Therefore $\bar{x} \in F^{\leq}\left(x^{*}\right)$ and $\bar{x} \in \bar{F} \leq\left(x^{*}\right)$.

From equation (11) and since $\lambda^{*} \geq 0, \lambda^{*} \neq 0, \mu^{*} \geq 0$ we have

$$
L\left(\bar{x}, \lambda^{*}, \mu^{*}\right)=\sum_{i=1}^{r} \lambda_{i}^{*} f_{i}(\bar{x})+\sum_{j=1}^{s} \mu_{j}^{*} g_{j}(\bar{x})
$$




$$
\begin{aligned}
& \leq \sum_{i=1}^{r} \lambda_{i}^{*} f_{i}(\bar{x}) \\
& <\sum_{i=1}^{r} \lambda_{i}^{*} f_{i}\left(x^{*}\right) .
\end{aligned}
$$

On the other hand,

$$
\begin{aligned}
\sum_{i=1}^{r} \lambda_{i}^{*} f_{i}\left(x^{*}\right) & =L\left(x^{*}, \lambda^{*}, 0\right) \\
& \leq L\left(x^{*}, \lambda^{*}, \mu^{*}\right) \\
& \leq L\left(\bar{x}, \lambda^{*}, \mu^{*}\right)
\end{aligned}
$$

which contradicts the observation above and completes the proof.

We remark that Theorem 4 is of theoretical interest since no constraint qualification, for example such as the Slater condition, is imposed. Interestingly, condition (11) is still necessary for a point $x^{*}$ to be Pareto even if $Q^{<}\left(x^{*}\right)$ is replaced by any nonempty subset $\mathcal{Q}\left(x^{*}\right)$ of $\mathcal{Q}$.

Corollary 3 Let a point $x^{*} \in F$ be a Pareto point for the (MOP) and assume that $\mathcal{Q}\left(x^{*}\right) \subset \mathcal{Q}$ is a nonempty set. Then there exist $0 \neq \lambda^{*} \geq$ $0, \mu^{*} \geq 0$ such that (11) holds for

$$
L(x, \lambda, \mu)=\sum_{i \in Q\left(x^{*}\right)} \lambda_{i} f_{i}(x)+\sum_{j \in M<\left(x^{*}\right)} \mu_{j} g_{j}(x)
$$

for all $\mu \geq 0$ and $x \in \bar{F} \leq\left(x^{*}\right)$ defined as

$$
\begin{aligned}
\bar{F}^{\leq}\left(x^{*}\right):= & \left\{x \in \mathbb{R}^{n}: f_{i}(x) \leq f_{i}\left(x^{*}\right) \forall i \in \mathcal{Q} \backslash \mathcal{Q}\left(x^{*}\right)\right\} \\
\cap & \left\{x \in \mathbb{R}^{n}: g_{j}(x) \leq 0 \forall j \in M^{=}\left(x^{*}\right)\right\} .
\end{aligned}
$$

\section{Proof:}

Follow the only if part of the proof of Theorem 4, replacing $\mathcal{Q}^{<}\left(x^{*}\right)$ by $\mathcal{Q}\left(x^{*}\right)$.

Corollary 4 Under the assumptions of Corollary 3 there exists an $i \in Q\left(x^{*}\right)$ such that $f_{i}\left(x^{*}\right) \leq f_{i}(x)$ for all $x \in \bar{F} \leq\left(x^{*}\right) \cap F$. 


\section{Proof:}

Based on Corollary 3, the left hand side inequality of (11) with (13) yields

$$
\sum_{j \in M^{<}\left(x^{*}\right)}\left(\mu_{j}-\mu_{j}^{*}\right) g_{j}\left(x^{*}\right) \leq 0 \quad \forall \mu \geq 0 .
$$

Let $\mu=0$, then we obtain

$$
\sum_{j \in M<\left(x^{*}\right)} \mu_{j}^{*} g_{j}\left(x^{*}\right) \geq 0
$$

which, due to the feasibility of $x^{*}$ and $\mu^{*} \geq 0$, results in

$$
\mu_{j}^{*} g_{j}\left(x^{*}\right)=0 \quad \forall j \in M^{<}\left(x^{*}\right) .
$$

Applying (14) to the right hand side of (11) with (13), we obtain

$$
\sum_{i \in Q\left(x^{*}\right)} \lambda_{i}^{*}\left(f_{i}\left(x^{*}\right)-f_{i}(x)\right)-\sum_{j \in M^{<}\left(x^{*}\right)} \mu_{j}^{*} g_{j}(x) \leq 0 \quad \forall x \in \bar{F}^{\leq}\left(x^{*}\right) .
$$

Since $x \in \bar{F} \leq\left(x^{*}\right) \cap F$ then $g_{j}(x) \leq 0$, which makes the right hand side term of (15) nonpositive. In order that (15) holds it must be that

$$
\exists i \in Q\left(x^{*}\right): f_{i}\left(x^{*}\right) \leq f_{i}(x) \text { for all } x \in \bar{F}^{\leq}\left(x^{*}\right) \cap F .
$$

Example 2 We illustrate the corollaries by a graphical example in Figure 2. Here we have three objective functions and two constraints. Figure 2 displays the feasible set $F$ as the shaded region and the rectangular level sets of the three objective functions. The boundaries of the level sets determine the corresponding level curves.

Consider first $x^{*}=B$ that is Pareto and its level curve of $f_{3}$ (the broken-line rectangle). We have $\mathcal{Q}^{<}\left(x^{*}\right)=\emptyset$ and $M^{<}\left(x^{*}\right)=\{2\}$. Let $f_{3}$ be of special interest, i.e. $\mathcal{Q}\left(x^{*}\right)=\{3\}$. Then $\bar{F} \leq\left(x^{*}\right)$ is the line segment $\overline{A B}$ connecting points $A$ and $B$. The corresponding Lagrangian function is $L=\lambda_{3} f_{3}+\mu_{2} g_{2}$ and it is easy to check that for point $B$ both inequalities in (11) hold, as Corollary 3 states. On the other hand, let $x^{*}=A$ that is not Pareto. We have $\mathcal{Q}^{<}\left(x^{*}\right)=\{3\}$ and $M^{<}\left(x^{*}\right)=\{2\}$. The Lagrangian function is again $L=\lambda_{3} f_{3}+\mu_{2} g_{2}$. The left hand side inequality of (11) holds but checking the right hand side inequality yields

$$
f_{3}\left(x^{*}\right) \leq f_{3}(x) \quad \forall x \in \overline{A B},
$$




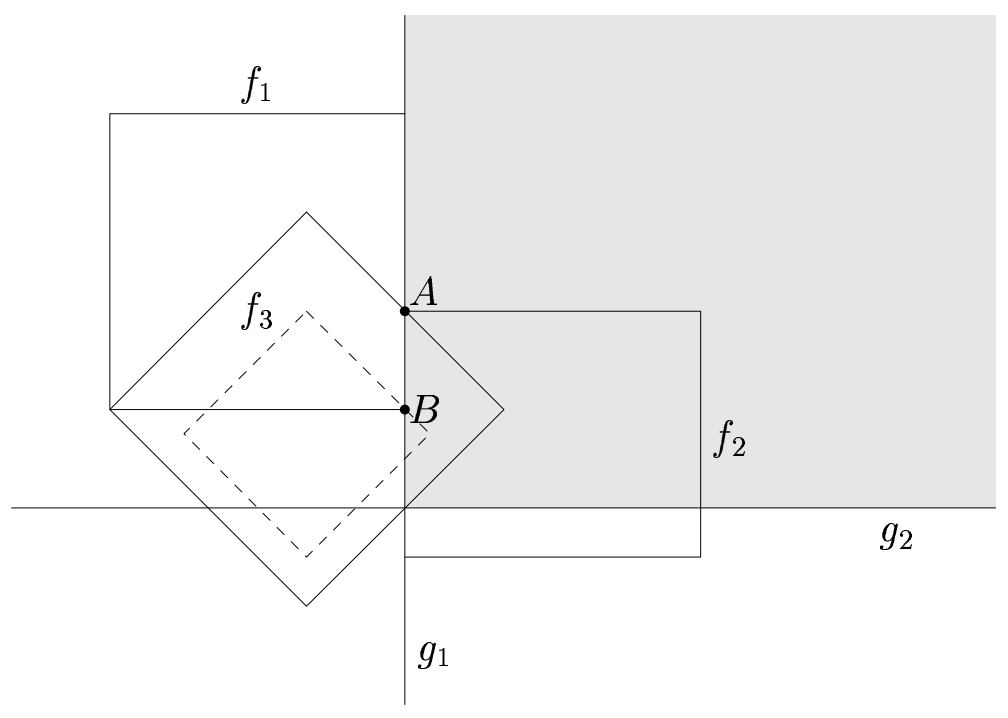

Figure 2: Illustration of Corollaries 3 and 4

which does not hold. Observe in Figure 2 that moving from $A$ to $B$ decreases the value of $f_{3}$ while the values of the other two objective functions remain unchanged.

Our main results of this section show that if $x^{*}$ is a Pareto point, then both $\mathcal{Q}^{<}\left(x^{*}\right)=\emptyset$ and the saddle point condition (11) holds. However, in a general decision making situation, given a solution $\hat{x} \in F$, we may not know whether it is a Pareto point. Assuming that it is not, we may want to improve some objective functions that are of special importance to us. In this context, Corollaries 3 and 4 become significant as they can support the decision process. Let $\mathcal{Q}(\hat{x})$ be the set of indices of the objective functions we would like to improve. We define $\bar{F} \leq(\hat{x})$ as:

$$
\begin{aligned}
& \bar{F}^{\leq}(\hat{x}):=\left\{x \in \mathbb{R}^{n}: f_{i}(x) \leq f_{i}(\hat{x}) \forall i \in \mathcal{Q} \backslash \mathcal{Q}(\hat{x})\right\} \\
& \cap \quad\left\{x \in \mathbb{R}^{n}: g_{j}(x) \leq 0 \forall j \in M^{=}(\hat{x})\right\} .
\end{aligned}
$$

and check whether the Lagrangian function $L(x, \lambda, \mu)$ has a saddle point for all $\mu \geq 0$ and for all $x \in \bar{F} \leq(\hat{x})$. If it does not, then $\hat{x}$ is not Pareto and we can in fact improve the chosen objective functions.

On the other hand, if $\hat{x}$ is a Pareto point, then based on Corollary 4, we can always find at least one objective function such that its value at $\hat{x}$ is 
the smallest for all $x \in \bar{F} \leq(\hat{x}) \cap F$, which indicates that at least one of the objective functions we have chosen cannot be improved and therefore should leave $\mathcal{Q}(\hat{x})$.

\subsection{A Saddle Point Result for the Infeasible Point Partition}

In this section we consider the infeasible point partitions rather than feasible point partitions, that is we use the index set $\mathcal{Q}_{0}^{<}\left(x^{*}\right)$ and $M_{0}^{<}\left(x^{*}\right)$ instead of $\mathcal{Q}^{<}\left(x^{*}\right)$ and $M^{<}\left(x^{*}\right)$, and examine whether the saddle point characterization still holds. As we follow upon the results of the previous section, we present new results without proofs. We start with a result similar to Lemma 1, which is only a sufficient condition for a point not to be Pareto.

Lemma 3 A point $x^{*} \in F$ is not a Pareto point for the (MOP) if both $\mathcal{Q}_{0}^{<}\left(x^{*}\right) \neq \emptyset$ and there exists a point $\bar{x} \in F$ such that

$$
\begin{aligned}
& f_{i}(\bar{x})<f_{i}\left(x^{*}\right) \quad \forall i \in \mathcal{Q}_{0}^{<}\left(x^{*}\right) \\
& f_{i}(\bar{x})=f_{i}\left(x^{*}\right) \quad \forall i \in \mathcal{Q}_{0}^{=}\left(x^{*}\right) .
\end{aligned}
$$

The lemma follows from the definition of Pareto solutions. For the converse it is only possible to show that if $x^{*}$ is not a Pareto point then $\mathcal{Q}_{0}^{<}\left(x^{*}\right)$ is not empty and there exists a point $\bar{x} \in \mathbb{R}^{n}$ for which the two conditions above hold, however the feasibility of $\bar{x}$ does not follow, due to the definition of $F_{0}^{\leq}\left(x^{*}\right)$.

Including the infeasible point partition of the index set of constraints leads us to Lemma 4 that corresponds to Lemma 2. Similarly to the previous lemma, Lemma 4 is only a sufficient condition.

Lemma $4 A$ point $x^{*} \in F$ is not a Pareto point for the (MOP) if both $\mathcal{Q}_{0}^{<}\left(x^{*}\right) \neq \emptyset$ and there exists a point $\bar{x} \in \mathbb{R}^{n}$ such that

$$
\begin{aligned}
& f_{i}(\bar{x})<f_{i}\left(x^{*}\right) \quad \forall i \in \mathcal{Q}_{0}^{<}\left(x^{*}\right) \\
& f_{i}(\bar{x})=f_{i}\left(x^{*}\right) \quad \forall i \in \mathcal{Q}_{0}^{=}\left(x^{*}\right) \\
& g_{j}(\bar{x})<0 \quad \forall j \in M_{0}^{<}\left(x^{*}\right) \\
& g_{j}(\bar{x})=0 \quad \forall j \in M_{0}^{=}\left(x^{*}\right)
\end{aligned}
$$


We remark that in the context of Lemma 4 , it is equivalent to say that if $x^{*} \in F$ is a Pareto point, there does not exist a point $\bar{x}$ satisfying conditions (16) to $(19)$ or $Q_{0}^{<}\left(x^{*}\right)=\emptyset$.

Using the definition of $\mathcal{Q}_{0}^{<}\left(x^{*}\right)$ we obtain a result analogous to Corollary 2.

Corollary 5 If a point $x^{*} \in F$ is a Pareto point for the $(M O P)$ and $M \neq$ $M_{0}^{=}\left(x^{*}\right)$, then there exists a point $\bar{x} \in \mathbb{R}^{n}$ such that (17), (18) and (19) hold.

The proof follows the proof of Corollary 2. Note that Corollary 5 implies that the point $\hat{x}$ used in that proof is feasible, due to (18) and (19).

We now again define the Lagrangian function

$$
L_{0}(x, \lambda, \mu):=\sum_{i \in \mathcal{Q}_{0}^{<}\left(x^{*}\right)} \lambda_{i} f_{i}(x)+\sum_{j \in M_{0}^{<}\left(x^{*}\right)} \mu_{j} g_{j}(x) .
$$

and show that if $x^{*}$ is a Pareto point then there exist $\lambda^{*}$ and $\mu^{*}$ such that $\left(x^{*}, \lambda^{*}, \mu^{*}\right)$ is a saddle point of this function. Let $\bar{F}_{0}^{\leq}\left(x^{*}\right)$ denote the set $\bar{F}^{\leq}\left(x^{*}\right)$ defined in Section 3.1 with $Q^{<}\left(x^{*}\right)$ and $M^{<}\left(x^{*}\right)$ replaced by $Q_{0}^{<}\left(x^{*}\right)$ and $M_{0}^{<}\left(x^{*}\right)$, respectively.

Theorem 5 If $x^{*} \in \bar{F}_{0}^{\leq}\left(x^{*}\right)$ is a Pareto point for the (MOP) then there exist $\lambda^{*} \geq 0, \lambda^{*} \neq 0$ and $\mu^{*} \geq 0$ such that

$$
L_{0}\left(x^{*}, \lambda^{*}, \mu\right) \leq L_{0}\left(x^{*}, \lambda^{*}, \mu^{*}\right) \leq L_{0}\left(x, \lambda^{*}, \mu^{*}\right)
$$

holds for all $\mu \geq 0$ and for all $x \in \bar{F}_{0}^{\leq}\left(x^{*}\right)$.

The proof is identical to the 'only if' part of the proof of Theorem 4.

We emphasize that the the Lagrangian function defined above is much easier to handle than the one given in [14]. The sets $\mathcal{Q}_{0}^{<}\left(x^{*}\right)$ and $M_{0}^{<}\left(x^{*}\right)$ can be easily determined by just comparing the objective functions' values at $x^{*}$ with these functions' global minima while the index sets used in [14] require much more complicated calculations. According to our result, to check whether a point $x^{*}$ is possibly a Pareto point one has to find the index sets $\mathcal{Q}_{0}^{<}\left(x^{*}\right)$ and $M_{0}^{<}\left(x^{*}\right)$, which is relatively easy due to the convexity assumption, and check whether for an appropriate $\lambda^{*}$ and $\mu^{*}$ the saddle point condition (20) is satisfied. Furthermore, all possible candidate points for Pareto solutions can be found by means of condition (20). When formulated for the infeasible point partitions, Corollaries 3 and 4 remain valid and, consequently, all the resulting conclusions supporting decision making remain valid, too. 


\section{Nonconvex Problems}

In this section we drop the convexity assumptions of the previous section, consider the general (nonconvex) $(M O P)$ and discuss again the relationships between Pareto points and saddle points. As the linear Lagrangian (10) cannot be used for nonconvex problems, we associate with the problem $\left(C C\left(M O P, x^{*}\right)\right)$ the augmented Lagrangian defined for $x \in F, y \in \mathbb{R}^{Q}$ and $r>0$ :

$$
\begin{aligned}
L_{a}(x, y, r)=\sum_{q=1}^{Q} f_{q}(x) & +\sum_{q=1}^{Q}\left[y_{q} \max \left\{f_{q}(x)-f_{q}\left(x^{*}\right), \frac{-y_{q}}{2 r}\right\}\right. \\
& \left.+r\left(\max \left\{f_{q}(x)-f_{q}\left(x^{*}\right), \frac{-y_{q}}{2 r}\right\}\right)^{2}\right]
\end{aligned}
$$

where $x^{*} \in F$.

In order to prove the main results of this section, following Rockafellar, see [7], we make some assumptions about the problem $\left(C C\left(M O P, x^{*}\right)\right)$.

\section{Assumption 1}

Let the problem $\left(C C\left(M O P, x^{*}\right)\right)$ satisfy the quadratic growth condition (QGC), i.e. there exists an $r \geq 0$ such that $L_{a}(x, 0, r)$ is bounded below as a function of $x, x \in F$.

The QGC is certainly satisfied if all the functions $f_{q}$ are bounded below on $F$ and thus in particular, if $F$ is compact and all the functions are lower semicontinuous on $F$. For more details we refer to Rockafellar, [7].

\section{Assumption 2}

Let the problem $\left(C C\left(M O P, x^{*}\right)\right)$ be (lower) stable of degree 2 (SoD2), i.e. there exist

1. an open neighbourhood $U$ of the origin in $\mathbb{R}^{Q}$, and

2. a function $\pi: U \rightarrow \mathbb{R}^{1}$ of class $C^{2}$ such that

$$
p(u) \geq \pi(u) \text { for all } u \in U
$$

and

$$
p(0)=\pi(0)
$$


where $p(u): \mathbb{R}^{Q} \rightarrow \mathbb{R}^{1}$ is the perturbation function associated with the problem $\left(C C\left(M O P, x^{*}\right)\right)$ and defined as

$$
p(u)=\min _{x \in F}\left\{\sum_{q=1}^{Q} f_{q}(x): f_{q}(x)-f_{q}\left(x^{*}\right) \leq u_{q} q \in \mathcal{Q}, u \in \mathbb{R}^{Q}\right\} .
$$

We emphasize that Assumption 1 is rather technical and not constraining while Assumption 2 is stronger and related to the curvature of the original objective functions, which should allow that the perturbation function $p(u)$ of the problem $\left(C C\left(M O P, x^{*}\right)\right)$ be supported by a function $\pi$. With these assumptions we can prove the existence of a Pareto-related saddle point.

Theorem 6 Let the problem $\left(C C\left(M O P, x^{*}\right)\right)$ satisfy $Q G C$ and be SoD2. Then $x^{*} \in F$ is a Pareto point for the $(M O P)$ if and only if there exist $\left(y^{*}, r^{*}\right), r^{*}>0$ such that

$$
L_{a}\left(x^{*}, y, r\right) \leq L_{a}\left(x^{*}, y^{*}, r^{*}\right) \leq L_{a}\left(x, y^{*}, r^{*}\right)
$$

holds for all $x \in F$ and $y \in \mathbb{R}^{Q}, r>0$.

\section{Proof:}

According to Theorem $1, x^{*} \in F$ is a Pareto solution of the $(M O P)$ if and only if it is an optimal solution of the problem $\left(C C\left(M O P, x^{*}\right)\right)$. Applying to this problem the results by Rockafellar, [7, Corollary 5.2], we obtain the desired result.

Now we are going to proceed as in the convex case. If a point $x^{*}$ is feasible but not Pareto for the $(M O P)$, using Theorem 2 we can restrict ourselves to the problem $\left(\operatorname{MOP}\left(\mathcal{Q}^{<}\left(x^{*}\right)\right)\right)$ in order to find a Pareto point dominating $x^{*}$. If a Pareto point $\hat{x}$ for the problem $\left(\operatorname{MOP}\left(\mathcal{Q}^{<}\left(x^{*}\right)\right)\right)$ has been found, we know that this point is also Pareto for the original $(M O P)$. In order to find a Pareto point for the smaller problem we investigate its scalarization $\left(C C\left(\operatorname{MOP}\left(\mathcal{Q}^{<}\left(x^{*}\right)\right), \hat{x}\right)\right)$ :

$$
\begin{aligned}
\min \sum_{q \in Q^{<}\left(x^{*}\right)} f_{q}(x) & \\
\text { s.t. } f_{q}(x)-f_{q}(\hat{x}) & \leq 0, \quad q \in \mathcal{Q}^{<}\left(x^{*}\right) \\
x & \in F \leq\left(x^{*}\right),
\end{aligned}
$$


where $\hat{x} \in F^{\leq}\left(x^{*}\right)$ is an arbitrary feasible point of $\left(M O P\left(\mathcal{Q}^{<}\left(x^{*}\right)\right)\right)$. Again, by Theorem 1, there is a one-to-one correspondence between Pareto points of the smaller problem $\left(\operatorname{MOP}\left(\mathcal{Q}^{<}\left(x^{*}\right)\right)\right)$ and optimal solutions of its scalarization.

Because $\hat{x} \in F \leq\left(x^{*}\right)$ we have

$$
f_{q}(\hat{x}) \leq f_{q}\left(x^{*}\right) \forall i \in \mathcal{Q} .
$$

From (23) and (24) we get

$$
\left.\begin{array}{l}
f_{q}(x)-f_{q}(\hat{x}) \leq 0 \\
f_{q}(x)-f_{q}\left(x^{*}\right) \leq 0
\end{array}\right\} \forall q \in \mathcal{Q}^{<}\left(x^{*}\right)
$$

and

$$
f_{q}(x)-f_{q}\left(x^{*}\right) \leq 0 \quad \forall i \in \mathcal{Q}^{=}\left(x^{*}\right),
$$

which, together with (25), yields

$$
\begin{aligned}
f_{q}(x)-f_{q}(\hat{x}) & \leq 0 \quad \forall q \in \mathcal{Q}^{<}\left(x^{*}\right) \\
f_{q}(x)-f_{q}\left(x^{*}\right) & \leq 0 \quad \forall q \in Q^{=}\left(x^{*}\right)
\end{aligned}
$$

Now the problem $\left(C C\left(M O P\left(\mathcal{Q}^{<}\left(x^{*}\right)\right), \hat{x}\right)\right)$ becomes

$$
\begin{aligned}
\min \sum_{q \in \mathcal{Q}^{<}\left(x^{*}\right)} f_{q}(x) & \\
f_{q}(x)-f_{q}(\hat{x}) & \leq 0 \quad \forall q \in \mathcal{Q}^{<}\left(x^{*}\right) \\
f_{q}(x)-f_{q}\left(x^{*}\right) & \leq 0 \quad \forall q \in Q^{=}\left(x^{*}\right) \\
x & \in F .
\end{aligned}
$$

Let $p^{s}(u)$ be the perturbation function associated with problem (26):

$$
p^{s}(u)=\min _{x \in F}\left\{\sum_{q \in \mathcal{Q}^{<}\left(x^{*}\right)} f_{q}(x):\left[\begin{array}{ll}
f_{q}(x)-f_{q}(\hat{x}) & \forall q \in \mathcal{Q}^{<}\left(x^{*}\right) \\
f_{q}(x)-f_{q}\left(x^{*}\right) & \forall q \in \mathcal{Q}^{=}\left(x^{*}\right)
\end{array}\right] \leq u, u \in \mathbb{R}^{Q}\right\} .
$$

We show that problem (26) is SoD2 if the same is true for the original problem $(C C(M O P, \hat{x}))$.

Lemma 5 Let $x^{*} \in F$ be not a Pareto point for the (MOP). Then for all points $\hat{x} \in F \leq\left(x^{*}\right)$ such that the problem $(C C(M O P, \hat{x}))$ is SoD2, the problem $\left(C C\left(\operatorname{MOP}\left(\mathcal{Q}^{<}\left(x^{*}\right)\right), \hat{x}\right)\right)$ is also SoD2. 


\section{Proof:}

Let $p(u)$ be the perturbation function associated with the problem $(C C(M O P, \hat{x}))$ and let

$$
\pi^{s}(u):=\pi(u)-\sum_{q \in \mathcal{Q}=\left(x^{*}\right)}\left(f_{q}(\hat{x})+u_{q}\right)
$$

where $\pi(u)$ is defined as in Assumption 2 for the problem $(C C(M O P, \hat{x}))$ and

$$
p(u) \geq \pi(u) \quad \forall u \in U .
$$

From (27) and (28) we get

$$
p(u)-\sum_{q \in \mathcal{Q}^{=}\left(x^{*}\right)}\left(f_{q}(\hat{x})+u_{q}\right) \geq \pi^{s}(u) .
$$

Now we shall show that

$$
p(u)-\sum_{\mathcal{Q}^{=}\left(x^{*}\right)}\left(f_{q}(\hat{x})+u_{q}\right) \leq p^{s}(u)
$$

where $p^{s}(u)$ is the perturbation function associated with the scalarized problem $\left(C C\left(M O P\left(\mathcal{Q}^{<}\left(x^{*}\right)\right), \hat{x}\right)\right)$. By definition,

$$
\begin{aligned}
& p(u)=\min _{x \in F}\left\{\sum_{q \in \mathcal{Q}} f_{q}(x): f_{q}(x)-f_{q}(\hat{x}) \leq u_{q} \forall q \in \mathcal{Q}\right\} \\
& =\min _{x \in F}\left\{\sum_{q \in \mathcal{Q}<\left(x^{*}\right)} f_{q}(x)+\sum_{q \in \mathcal{Q}=\left(x^{*}\right)} f_{q}(x):\right. \\
& \left.\left[\begin{array}{ll}
f_{q}(x)-f_{q}(\hat{x}) & \forall q \in \mathcal{Q}^{<}\left(x^{*}\right) \\
f_{q}(x)-f_{q}(\hat{x}) & \forall q \in \mathcal{Q}^{=}\left(x^{*}\right)
\end{array}\right] \leq u\right\} .
\end{aligned}
$$

Observe that

$$
f_{q}(\hat{x})=f_{q}\left(x^{*}\right) \text { for all } q \in \mathcal{Q}^{=}\left(x^{*}\right)
$$

Therefore

$$
p(u)=\min _{x \in F}\left\{\sum_{q \in \mathcal{Q}^{<}\left(x^{*}\right)} f_{q}(x)+\sum_{q \in \mathcal{Q}=\left(x^{*}\right)} f_{q}(x):\right.
$$




$$
\begin{aligned}
& {\left.\left[\begin{array}{ll}
f_{q}(x)-f_{q}(\hat{x}) & \forall q \in \mathcal{Q}^{<}\left(x^{*}\right) \\
f_{q}(x)-f_{q}\left(x^{*}\right) & \forall q \in \mathcal{Q}^{=}\left(x^{*}\right)
\end{array}\right] \leq u\right\} } \\
& \leq \min _{x \in F}\left\{\begin{array}{l}
\sum_{q \in \mathcal{Q}^{<}\left(x^{*}\right)} f_{q}(x)+\sum_{q \in \mathcal{Q}=\left(x^{*}\right)}\left(f_{q}(\hat{x})+u_{q}\right): \\
\left.\left[\begin{array}{ll}
f_{q}(x)-f_{q}(\hat{x}) & \forall q \in \mathcal{Q}^{<}\left(x^{*}\right) \\
f_{q}(x)-f_{q}\left(x^{*}\right) & \forall q \in \mathcal{Q}^{=}\left(x^{*}\right)
\end{array}\right] \leq u\right\}
\end{array}\right. \\
&\left.=\min _{x \in F}\left\{\begin{array}{ll}
\sum_{q \in \mathcal{Q}^{<}\left(x^{*}\right)} f_{q}(x):\left[\begin{array}{ll}
f_{q}(x)-f_{q}(\hat{x}) \\
f_{q}(x)-f_{q}\left(x^{*}\right)
\end{array} \quad \forall q \in \mathcal{Q}^{<}\left(x^{*}\right)\right. \\
+ & \sum_{q \in \mathcal{Q}=\left(x^{*}\right)}\left(f_{q}(\hat{x})+u_{q}\right)
\end{array}\right] \leq u\right\} \\
&=p^{s}(u)+\sum_{q \in \mathcal{Q}^{=}\left(x^{*}\right)}\left(f_{q}(\hat{x})+u_{q}\right) .
\end{aligned}
$$

The inequality above results from the condition $f_{q}(x)-f_{q}\left(x^{*}\right) \leq u_{q} \forall q \in$ $\mathcal{Q}^{=}\left(x^{*}\right)$, after which the expression under the second sum becomes constant and the minimization is carried out with respect to the first sum. Clearly, (31) proves (30). Furthermore we shall show that $p^{s}(0)=\pi^{s}(0)$.

$$
\begin{aligned}
p(0)= & \min _{x \in F}\left\{\sum_{q \in \mathcal{Q}^{<}\left(x^{*}\right)} f_{q}(x)+\sum_{q \in \mathcal{Q}^{=}\left(x^{*}\right)} f_{q}(x):\right. \\
& \left.\quad\left[\begin{array}{c}
f_{q}(x)-f_{q}(\hat{x}) \quad \forall q \in \mathcal{Q}^{<}\left(x^{*}\right) \\
f_{q}(x)-f_{q}(\hat{x}) \quad \forall q \in \mathcal{Q}^{=}\left(x^{*}\right)
\end{array}\right] \leq 0\right\} \\
= & \min _{x \in F \leq\left(x^{*}\right)}\left\{\sum_{q \in \mathcal{Q}^{<}\left(x^{*}\right)} f_{q}(x): f_{q}(x)-f_{q}(\hat{x}) \leq 0 \forall q \in \mathcal{Q}^{<}\left(x^{*}\right)\right\} \\
& +\sum_{q \in \mathcal{Q}=\left(x^{*}\right)} f_{q}(\hat{x}) \\
= & \min _{x \in F}\left\{\sum_{q \in \mathcal{Q}^{<}\left(x^{*}\right)} f_{q}(x):\left[\begin{array}{ll}
f_{q}(x)-f_{q}(\hat{x}) & \left.\forall q \in \mathcal{Q}^{<}\left(x^{*}\right)\right] \leq 0 \\
f_{q}(x)-f_{q}\left(x^{*}\right) \quad \forall q \in \mathcal{Q}^{=}\left(x^{*}\right)
\end{array}\right] \leq\right. \\
& +\sum_{q \in \mathcal{Q}^{=}\left(x^{*}\right)} f_{q}(\hat{x}) \\
= & p^{s}(0)+\sum_{q \in \mathcal{Q}^{=}\left(x^{*}\right)} f_{q}(\hat{x}) .
\end{aligned}
$$


The second equality follows from the definition of $F \leq\left(x^{*}\right)$ and $f_{q}(x)=f_{q}\left(x^{*}\right)$ for all $x \in F \leq\left(x^{*}\right)$ and all $q \in \mathcal{Q}^{=}\left(x^{*}\right)$. Now we apply the definition of $F \leq\left(x^{*}\right)$ again and get the third equality.

Since $p(0)=\pi(0)=\pi^{s}(0)+\sum_{q \in \mathcal{Q}=\left(x^{*}\right)} f_{q}(\hat{x})$ we obtain the desired result.

With Lemma 5 we can prove the main theorem of this section.

Theorem 7 Let $x^{*} \in F$ be not a Pareto solution for the (MOP). Let the problem $(C C(M O P, \hat{x}))$ satisfy the $Q G C$ and be SoD2. If $(\hat{x}, \hat{y}, \hat{r})$ is a saddle point of the augmented Lagrangian function associated with the problem $\left(C C\left(\operatorname{MOP}\left(\mathcal{Q}^{<}\left(x^{*}\right)\right), \hat{x}\right)\right)$, then there exist $\left(y^{*}, r^{*}\right), r^{*}>0$, such that $\left(\hat{x}, y^{*}, r^{*}\right)$ is a saddle point of the augmented Lagrangian function associated with the problem $(C C(M O P, \hat{x}))$.

\section{Proof:}

Since the problem $(C C(M O P, \hat{x}))$ satisfies the $\mathrm{QGC}$ so does the problem $\left(C C\left(M O P\left(\mathcal{Q}^{<}\left(x^{*}\right), \hat{x}\right)\right)\right)$. From Lemma 5, problem $\left(C C\left(\operatorname{MOP}\left(\mathcal{Q}^{<}\left(x^{*}\right), \hat{x}\right)\right)\right)$ is SoD2. From Theorem 6, $\hat{x}$ is a Pareto point for problem $\left(\operatorname{MOP}\left(\mathcal{Q}^{<}\left(x^{*}\right)\right)\right.$, and from Theorem 2, $\hat{x}$ is also Pareto for the $(M O P)$. Therefore, due to Theorem 6 again, the result follows.

\section{Conclusions}

In this paper, Pareto solutions of multiple objective programs (MOPs) are related to saddle points of some Lagrangian-type scalarizing functions. The common foundation for all the results is determined by Charnes and Cooper's scalarization of MOPs as well as by the partitions of the set of objective functions and the set of constraints introduced in this paper. As the partitions distinguish the objective functions whose values can be still improved, they are of special significance in decision making. Saddle point characterizations of Pareto points for convex and nonconvex MOPs are derived and their usefulness in decision making is discussed.

The results could be extended in several directions. First of all, other scalarizations of MOPs, such as the $\epsilon$-constraint scalarization (see [1]) or the weighted Tchebycheff scalarization (see [3]) could be used and lead to other Lagrangian functions whose saddle points would be checked. Furthermore, 
assuming that the scalarized version of the original (MOP) takes the form of a nonlinear program with equality constraints, a simpler augmented Lagrangian function could be applied (see [7]).

\section{References}

[1] V. Chankong and Y. Y. Haimes. Multiobjective Decision Mmaking: Theory and Methodology. North Holland, New York, 1983.

[2] A. Charnes and W. Cooper. Management Models and Industrial Applications of Linear Programming. John Wiley and Sons, New York, 1961.

[3] Steuer R. E. Multiple Criteria Optimization: Theory, Computation and Application. Wiley, New York, 1986.

[4] M. Ehrgott. Multiple Criteria Optimization - Classification and Methodology. Shaker Verlag, Aachen, 1997. Ph.D. Dissertation, University of Kaiserslautern.

[5] M. Ehrgott, H.W. Hamacher, K. Klamroth, S. Nickel, A. Schöbel, and M.M. Wiecek. A note on the equivalence of balance points and Pareto solutions in multiple-objective programming. Journal of Optimization Theory and Applications, 92(1):209-212, 1997.

[6] P. Iacob. Saddle point duality theorems for Pareto optimization. L'Analyse Numerique et la Theorie de l'Approximation, 15(1):37-40, 1986.

[7] R.T. Rockafellar. Augmented Lagrange multiplier functions and duality in nonconvex programming. SIAM Journal on Control, 12(2):268-285, 1974 .

[8] Y. Sawaragi, H. Nakayama, and T. Tanino. Theory of Multiobjective Optimization. Academic Press, Orlando, 1985.

[9] C. Singh, D. Bhatia, and N. Rueda. Duality in nonlinear multiobjective programming using augmented Lagrangian functions. Journal of Optimization Theory and Applications, 88(3):659-670, 1996. 
[10] M. L. TenHuisen and M. M. Wiecek. An augmented Lagrangian scalarization for multiple objective programming. Proceedings of the MOPGP‘96 Conference, Malaga, Spain. to appear 1998.

[11] M. L. TenHuisen and M. M. Wiecek. Vector optimization and generalized Lagrangian duality. Annals of Operations Research, 51:15-32, 1994.

[12] M. L. TenHuisen and M. M. Wiecek. Efficiency and solution approaches to bicriteria nonconvex programs. Journal of Global Optimization, 11:225-251, 1997.

[13] I. Valyi. Approximate saddle-point theorems in vector optimization. Journal of Optimization Theory and Applications, 55(3):435-448, 1987.

[14] M. van Rooyen, X. Zhou, and S. Zlobec. A saddle-point characterization of Pareto optima. Mathematical Programming, 67:77-88, 1994. 